\title{
Abordaje del paciente pediátrico con neutropenia febril y enfermedad oncológica
}

\author{
María Paula González-Galvis* \\ Luis Miguel Sosa-Ávila** \\ Ernesto Rueda-Arenas ${ }^{* * *}$
}

\begin{abstract}
*Residente de III año de Pediatría. Departamento de Pediatría. Escuela de Medicina. Facultad de Salud. Universidad Industrial de Santander. Bucaramanga. Santander. Colombia.

**Médico Infectólogo Pediatra. Profesor planta. Departamento de Pediatría. Universidad Industrial de Santander. Bucaramanga. Santander. Colombia. ***Médico Hemato-oncólogo Pediatra. Profesor planta. Departamento de Pediatría. Universidad Industrial de Santander. Bucaramanga. Santander. Colombia.
\end{abstract}

Correspondencia: Dra. María Paula González Galvis. Dirección: Carrera 33 \# 28- 126. Departamento de Pediatría. Hospital Universitario de Santander. Bucaramanga. Santander. Colombia. Correo electrónico: m-paulag@hotmail.com.

\section{RESUMEN}

Introducción: La neutropenia febril en el paciente pediátrico con enfermedad oncológica es una situación frecuente asociada a la quimioterapia que lleva a una alta morbimortalidad. Su principal etiología es la infección, ameritando un estudio oportuno y completo para identificar el origen e instaurar un tratamiento de forma rápida y dirigida, por lo cual es necesario conocer sobre esta patología y su abordaje. Objetivo: Realizar una revisión actual y completa de esta patología, abordando su epidemiología, etiología, diagnóstico clínico y tratamiento. Metodología de búsqueda: Se realizó una búsqueda en bases de datos como Cochrane Library, Medscape, Pubmed, RIMA, Ebscohost, HINARI, Dynamed, NEJM, usando palabras clave como neutropenia, fiebre, infección, riesgo, neoplasia y tratamiento. Se seleccionaron principalmente guías de práctica clínica, revisiones sistemáticas y estudios clínicos aleatorios. Discusión: Esta situación se presenta principalmente en pacientes con neoplasias hematolinfoides y la causa de la fiebre se debe en primer lugar a una infección, cuyo agente etiológico varía según la procedencia del caso, el uso de catéteres intravenosos o si hay neutropenia profunda y prolongada. El abordaje diagnostico debe ser mediante un examen físico minucioso y el uso de herramientas como hemograma, reactantes de fase aguda y policultivos. El uso de modelos pronósticos para realizar estratificación del riesgo de complicaciones permite dirigir el manejo antibiótico que se va a instaurar. Conclusión: Mediante la mejoría del conocimiento de esta enfermedad, las estrategias de diagnóstico y tratamiento han podido mejorar la sobrevida y el pronóstico de los pacientes con esta patología. MÉD UIS. 2015;28(3):353-62.

Palabras clave: Fiebre. Neutropenia. Neoplasias. Pediatría. Infección.

\section{Approach to the pediatric patient with febrile neutropenia and oncologic disease}

\section{ABSTRACT}

Introduction: Febrile neutropenia in pediatric patients with oncological disease, is a common condition leading to high morbidity and mortality. The main cause is infection, meriting a timely and comprehensive study to identify its source and begin an early and a targeted therapy, it is necessary to know about this disease and its management. Objective: Perform a current review of this pathology by addressing its epidemiology, etiology, clinical diagnosis and treatment. Methods: Search in database like Cochrane Library, Medscape, Pubmed, RIMA, Ebscohost, HINARI, Dynamed, NEJM, using keywords like neutropenia, fever, infection, risk, neoplasia and treatment. We selected clinical practice guidelines, systematic reviews and randomized clinical trials. Discussion: It is a common situation mainly in patients with hematological malignancy and the cause of the fever is due to an infection whose etiologic agent varies according to origin of the case, use of intravenous catheters or prolonged and deep neutropenia. The diagnostic approach must be through physical examination and the use of diagnostic tools such blood count, acute phase reactants and polyculture. The use of prognostic models for risk stratification allows to guide the antibiotic treatment. Conclusion: Improving the knowledge of this disease, the diagnosis and treatment strategies, it has been able to improve survival and prognosis of this disease. MÉD UIS. 2015;28(3):353-62.

Keywords: Fever. Neutropenia. Neoplasms. Pediatrics. Infection. 
¿Cómo citar este artículo?: González-Galvis MP, Sosa-Ávila LM, Rueda-Arenas E. Abordaje del paciente pediátrico con neutropenia febril y enfermedad oncológica. MÉD UIS. 2015;28(3):353-62.

\section{INTRODUCCIÓN}

Las enfermedades oncológicas representan un importante problema de salud en todo el mundo, en Estados Unidos la incidencia anual de cáncer pediátrico asciende a 137 casos por un millón de habitantes', en Argentina se estiman 12 a 14 casos por cada 100000 niños menores de 15 años de $\operatorname{edad}^{2}$ y en el caso del área metropolitana de Bucaramanga, Colombia, 140 casos por un millón de habitantes en menores de 15 años'. Hasta el $60 \%$ de los pacientes neutropénicos que tienen fiebre presentan una infección ${ }^{3}$ y el $16 \%$ de las muertes a los cinco años del diagnóstico son por complicaciones de la quimioterapia, principalmente la infección ${ }^{4}$. Una década atrás la letalidad del paciente pediátrico con neutropenia febril era del $10 \%$, actualmente se reporta una frecuencia que oscila entre $2 \%$ a $6 \%$ en niños ${ }^{5,6}$.

El paciente oncológico se encuentra más expuesto a padecer infecciones debido a su estado de inmunosupresión, ya sea por los efectos producidos por su enfermedad de base o por el manejo instaurado para la misma ${ }^{7}$. Durante el tratamiento antineoplásico los agentes citotóxicos son administrados junto con otras terapias inmunosupresoras ${ }^{7,8}$, lo que produce efectos secundarios múltiples en el organismo, creando alteraciones en el sistema inmune, tanto innato como adaptativo y favoreciendo la invasión por diferentes microorganismos. A nivel del sistema innato, se rompe la barrera cutánea con el uso de catéteres, se produce inflamación y lesión de las mucosas debido a la quimioterapia lo cual permite la invasión de agentes patógenos. Adicionalmente, uno de los principales efectos es el compromiso a nivel de los neutrófilos, monocitos circulantes y macrófagos tisulares, en donde los agentes citotóxicos causan una alteración en la quimiotaxis, en la función bactericida, en la producción de agentes superóxidos y causan la disminución en el número de neutrófilos y monocitos; siendo así, la neutropenia una situación frecuente en estos pacientes. El resultado de estas alteraciones cuantitativas $y$ funcionales es la incapacidad del sistema inmune de responder adecuadamente a la invasión por patógenos bacterianos y fúngicos, aumentando por tanto el riesgo de infección?.
Debido a la reacción inflamatoria limitada que presentan estos pacientes la fiebre puede ser la única manifestación de una infección grave ${ }^{4,10}$, razón por la cual esta entidad en el paciente neutropénico es una situación que debe considerarse como una urgencia médica que amerita un estudio oportuno y completo. Es fundamental que los médicos en su práctica clínica detecten de forma temprana al paciente oncológico con neutropenia y fiebre y dominen las diferentes estrategias de abordaje diagnóstico y terapéutico de esta patología. Con tal fin, el objetivo de este articulo es realizar una revisión actual y completa de esta patología, abordando su definición, epidemiología, etiología, diagnóstico clínico y tratamiento.

\section{Metodología de Búsqueda}

Se realizó una búsqueda en base de datos como Cochrane Library, Medscape, Pubmed, RIMA, Ebscohost, HINARI, Dynamed, NEJM usando palabras clave como neutropenia, fiebre, infección, riesgo, neoplasia y tratamiento. Se identificaron artículos originales, monografías, guías de práctica clínica y revisiones sistemáticas en español e inglés publicados después de 1996, que incluyeran dentro de la publicación características sobre epidemiología de la enfermedad, aproximación diagnóstica, predictores de alto riesgo y abordaje terapéutico y se excluyeron aquellos que carecían de soporte científico. En total fueron 37 publicaciones las seleccionadas.

\section{Definiciones}

Teniendo en cuenta la progresión rápida de este cuadro y su alta morbimortalidad, es importante detectar rápidamente esta patología, por eso las siguientes definiciones permitirán identificar aquellos pacientes que en el contexto de un cuadro oncológico presentan neutropenia y fiebre.

\section{FIEBRE}

Se define como el registro de temperatura axilar de $38,3^{\circ} \mathrm{C}$ o mayor en una toma, o una temperatura de $38^{\circ} \mathrm{C}$ o mayor en dos medidas consecutivas separadas por una hora, o una temperatura mayor de $38,1^{\circ} \mathrm{C}$ al menos durante una hora 8,11 . A pesar de que la temperatura rectal tiene mayor sensibilidad, se 
prefiere la medición de la temperatura axilar teniendo en cuenta el riesgo potencial de translocación bacteriana si el paciente cursa con mucositis?

\section{NEUTROPENIA}

Se define como la reducción en el recuento absoluto de neutrófilos en la sangre por debajo de 1500 células $/ \mathrm{mm}^{3}$, la cual está determinada por la suma de segmentados y cayados. En la tabla 1 se puede observar la clasificación según el recuento celular, y teniendo en cuenta esta, se tomará como neutropenia severa un recuento de neutrófilos menor o igual a 500 células $/ \mathrm{mm}^{3}$ o conteo entre 500 y 1000 células $/ \mathrm{mm}^{3}$ con una caída brusca entre el $25 \%$ y $50 \%$ del valor en la última semana9.

Tabla 1. Clasificación neutropenia febril

\begin{tabular}{|l|c|}
\hline \multicolumn{1}{|c|}{ Neutropenia } & Conteo absoluto \\
\hline Leve & $1500-1000$ células $/ \mathrm{mm}^{3}$ \\
\hline Moderada & $1000-500$ células $/ \mathrm{mm}^{3}$ \\
\hline Severa & $<500$ células $/ \mathrm{mm}^{3}$ \\
\hline
\end{tabular}

Fuente: Del Pont JM, Casanueva E. Comité Nacional de Infectología Pediátrica. Consenso sobre el cuidado del paciente oncológico neutropénico febril. Arch Argent Pediatr. 2010;9:47-70.

Los conceptos anteriores son definiciones operativas que permiten al personal médico tomar decisiones que podrían disminuir la probabilidad de adquirir una infección grave; sin embargo, debe primar el criterio clínico teniendo en cuenta que muchos pacientes oncológicos pueden tener un alto riesgo de presentar una infección a pesar de tener conteos normales de neutrófilos debido a alteraciones no cuantitativas, sino cualitativas de los mismos, término al cual se le ha denominado neutropenia funcional ${ }^{12}$.

\section{EPIDEMIOLOGÍA DE LOS EPISODIOS FEBRILES EN EL} PACIENTE PEDIÁTRICO CON ENFERMEDAD ONCOLÓGICA

La fiebre es una complicación frecuente que amenaza la vida del paciente oncológico que recibe quimioterapia ${ }^{13}$; se ha identificado que más del $80 \%$ de los pacientes con malignidades hematológicas y de $10 \%$ a $50 \%$ de los pacientes con tumores sólidos desarrollarán fiebre asociado a neutropenia al recibir más de un ciclo de quimioterapia ${ }^{12}$. La neutropenia febril es la segunda causa de ingreso hospitalario en oncología pediátrica después de las admisiones para quimioterapia, prolonga la estancia hospitalaria hasta por 13 días ${ }^{10,14}$, incrementa los costos y afecta la calidad de vida del paciente.
La neutropenia febril es más común en pacientes con neoplasias hematolinfoides ${ }^{11,12,15}$. En estudios norteamericanos se encuentra que hasta el $34 \%$ de los casos de neutropenia febril corresponden a pacientes con leucemia linfoide aguda, seguido de leucemia mieloide aguda con un $11 \%$ y linfoma no Hodgkin con un 9\%. Otros tumores en los cuales se ha reportado esta entidad son las neoplasias del sistema nervioso central en el $6 \%$, riñón en el 3\% y tumores gonadales ${ }^{16}$. Estudios realizados en Colombia muestran cifras similares, encontrando que el $47 \%$ de los casos de neutropenia febril corresponden a pacientes con leucemia linfoide aguda, linfoma no Hodgkin en el 21\%, leucemia mieloide aguda en el $10 \%$ y menos frecuentemente en el tumor de Wilms, retinoblastoma, rabdomiosarcoma y tumores del sistema nervioso central ${ }^{15}$. En un estudio realizado en el Hospital Universitario de Santander en el periodo comprendido entre enero del $2007 \mathrm{y}$ enero 2008 , el $78 \%$ de los episodios de neutropenia febril correspondieron a pacientes con neoplasia hematolinfoide ${ }^{11}$.

\section{ETIOLOGÍA}

Al analizar la causa de la fiebre, los estudios indican que la mayoría de los casos son secundarios a una infección $3,13,15$; pudiendo ser de origen viral, fúngica o bacteriana, siendo la infección bacteriana la complicación más frecuente con presentación más temprana durante el episodio febril en un paciente neutropénico ${ }^{2}$. La identificación del origen de la infección se logra solo en el 30\% a 50\% de los casos ${ }^{15,17}$, y cuando se reconoce el foco, este generalmente corresponde a infecciones del tracto respiratorio superior e inferior como neumonía, sinusitis y otitis, estas dos últimas en menor frecuencia3,15,18. También se han reportado casos a nivel de sistema gastrointestinal con infecciones que varían desde gastroenteritis, mucositis y esofagitis, hasta enterocolitis neutropénica o tiflitis. Otros lugares identificados, corresponden a infecciones de piel y tejido blando, y menos común las infecciones del sistema nervioso central como la meningitis ${ }^{2}$. La bacteremia se reporta con una incidencia del 10\% al $24 \%$ de los $\operatorname{casos}^{14}$.

Estudios nacionales muestran estadísticas similares a las presentadas con anterioridad. En un estudio descriptivo realizado en 1999 en el centro hematológico infantil de la Universidad de Antioquia ${ }^{15}$, se reportó como foco de infección principal la bronconeumonía en el $81,8 \%$ de los pacientes, 
mucositis entre $15 \%$ al $35 \%$, infección de tejido blando en el $4,5 \%$, enfermedad diarreíca aguda también en el $4,5 \%$ y otros como sinusitis, osteomielitis y flebitis ${ }^{15}$, lo cual varía un poco de lo encontrado en el Hospital Universitario de Santander, donde la enfermedad diarreíca aguda es el foco en el $15 \%$, mucositis y tiflitis en el $12 \%$, infección de tejidos blandos en el $10 \%$, neumonía en el $7 \%$ e infección de vías urinarias en el 2,5\% ${ }^{11}$. El sistema urinario es una foco de infección importante reportándose una frecuencia de hasta el $26 \%$ en la literatura mundial $6,17,19$, con menor frecuencia en estudios locales $(2,5 \%)^{11}$; encontrandose que cuando se han aislado en hemocultivos gérmenes Gram negativos y hongos, la gran mayoría de casos se relacionan con infecciones del tracto urinario ${ }^{18,19}$.
El aislamiento del germen es importante para definir un manejo dirigido y obtener mejores respuestas a la terapéutica instaurada; no obstante, en la mayoría de los casos solo se puede aislar el agente etiológico responsable en el $10 \%$ al $30 \%$ de los $\operatorname{casos}^{2,7}$, cifra que aumenta hasta el $40 \%$ en pacientes que además cursan con sepsis al usar métodos de aislamiento convencional como los cultivos. En la Tabla 2 se especifican los patógenos más frecuentes según el tipo de organismo. Dentro de los gérmenes detectados con mayor frecuencia en pacientes con neutropenia febril de alto riesgo estan S. aureus, E. faecalis, S. pneumoniae, S. pyogenes, E. coli, Klebsiella pneumoniae, Acinetobacter y $P$. aeruginosa $a^{5,20,21}$, esta última se ha reportado hasta en un $5 \%$ de los casos en niños.

Tabla 2. Agentes etiológicos más frecuentes en el paciente neutropenico febril

\begin{tabular}{|l|l|l|l|}
\hline \multicolumn{2}{|c|}{ BACTERIAS } & \multicolumn{1}{c|}{ VIRUS } & \multicolumn{1}{c|}{ HONGOS } \\
\hline \multicolumn{1}{|c|}{ GRAM POSITIVO } & \multicolumn{1}{|c|}{ GRAM NEGATIVOS } & & \multirow{2}{*}{ Cándida } \\
S. aureus & E. coli & Herpes 1 Y 2 & Aspergillus \\
S. epidermidis & Klebsiella & Citomegalovirus & Mucor \\
S. alfa hemolítico & Pseudomonas & Virus Epstein Barr & Varicela zoster \\
Enterococo & Serratia & & \\
S. Pneumoniae & Enterobacter & & \\
L. Monocytogenes & Acinetobacter & Salmonella & \\
\hline
\end{tabular}

Fuente: Matloob M, Fadoo Z. Febrile neutropenia in pediatric cancer patients: Experience from a tertiary health care facility of Pakistan. Pediatric infectious disease. 2014,6:89-93.

En las últimas décadas, se ha visto un cambio en la epidemiología de las infecciones en pacientes con neutropenia febril, debido a modificaciones en los tratamientos quimioterapéuticos con manejos más mieloablativos que llevan a mayor intensidad y duración de la neutropenia, aumento del tiempo de la internación y por consiguiente, incremento de las infecciones por gérmenes nosocomiales². Entre los factores de riesgo para tener una infección por gérmenes Gram positivos se incluye la mucositis inducida por quimioterapia, la profiláxis antibiótica ${ }^{21}$ y el uso cada vez mayor de catéteres intravenosos, este último relacionado principalmente con $S$. aureus².

Otros factores relacionados con el cambio en la microbiología de la infección es la procedencia del caso; por ejemplo, en países desarrollados los organismos Gram positivos son los más frecuentemente aislados $(57 \%)^{5,21}$, reemplazando a los gérmenes Gram negativos (34\%), con poca prevalencia de aislamientos polimicrobianos $(9 \%)^{21}$. Por otra parte en países de bajos ingresos, los datos reportados son diversos, con gran frecuencia de gérmenes Gram negativos y asilamientos polimicrobianos $s^{6,11}$.

La infección fúngica invasiva se presenta con una frecuencia hasta del $20 \%$, 22 , y un factor de riesgo importante para su presentación es la neutropenia profunda y prolongada la cual es definida por un conteo de neutrófilos menor de 100 por más de siete días ${ }^{12}$. La tasa de confirmación de este tipo de patógenos es solo del 10\%10,22. Especies de cándida como albicans, parapsilosis, tropicalis, son las más comúnmente aisladas dentro del grupo de los hongos, seguido por Aspergillus spp principalmente en pacientes con neutropenia prolongada por más de dos semanas ${ }^{10,11}$ y algunos estudios indican que en casos de infección fúngica documentada, la orina fue el sitio más común de aislamiento ${ }^{18}$. Entre otros gérmenes relacionados, se reporta que de los pacientes pediátricos que reciben quimioterapia, del 
$15 \%$ al 20\% desarrollarán una infección por $P$. jiroveci si no reciben profilaxis. Así mismo, entre el grupo de virus son comunes las reactivaciones de virus de herpes, citomegalovirus y varicela zoster ${ }^{9}$.

Es importante reconocer que la prevalencia de los patógenos varía mucho, inclusive entre los diferentes hospitales de una misma ciudad, por lo que se recomienda tener en cuenta la epidemiología (tipo de patógeno, patrones de resistencia y susceptibilidad) del centro hospitalario en el que se encuentre el médico tratante ${ }^{2}$.

\section{Aspectos Clínicos}

\section{ABORDAJE INICIAL}

La anamnesis completa y un examen físico minucioso son herramientas muy útiles, siendo pilares diagnósticos en el momento de evaluar al paciente febril y neutropénico ${ }^{10}$. Es necesario preguntar por potenciales exposiciones en casa y colegio, determinar uso previo de antibióticos, comorbilidades de base y manifestaciones clínicas que guíen hacia un foco infeccioso en particular ${ }^{2}$. Siempre se debe examinar al paciente de pies a cabeza, con énfasis en las áreas de mayor riesgo de infección como son los oídos, la nariz y la garganta, debido a la posibilidad de infección por gérmenes como Aspergillus y Mucor. Adicionalmente, buscar lesiones en piel, teniendo en cuenta que la varicela y el herpes zoster pueden dar manifestaciones inespecíficas como dolor o hipoestesia o incluso no dar ninguna lesión". También se debe evaluar cuidadosamente el sistema respiratorio, buscar signos abdominales que hagan pensar en tiflitis, valorar la región perineal buscando signos de inflamación local y visualizar los trayectos de catéteres buscando signos inflamatorios 0 infecciosos.

No hay que olvidar que la reacción inflamatoria de estos pacientes es muy escasa, lo cual hace que las claves clínicas para identificar un foco de infección sean muy sutiles o incluso nulas, pudiendo ser la fiebre el único dato que indique que se está ante una infección grave ${ }^{21,23}$, es por esto que las herramientas diagnósticas de laboratorio son de gran utilidad en el paciente con neutropenia febril.

\section{EXÁMENES DIAGNÓSTICOS}

En todo paciente con neutropenia febril se recomienda solicitar, antes de iniciar el manejo antibiótico o de hospitalizar al paciente, un cuadro hemático con diferencial de leucocitos y plaquetas, creatinina, nitrógeno ureíco, electrolitos, transaminasas, proteína $C$ reactiva, parcial de orina y dos hemocultivos. En caso de tener catéter central, se realizará toma simultánea, una central y otra periférica, y en caso de no tenerlo se tomarán dos muestras periféricas separadas $2,10,12$.

Para estudiar la infección de la vía urinaria, en algunos protocolos solo se realiza el examen general de orina, mientras que el urocultivo se recomienda para los pacientes que presenten síntomas urinarios o exista un resultado patológico de un parcial de orina o el paciente tenga un catéter vesical intermitente o permanente ${ }^{10,12,23}$. Sin embargo, teniendo en cuenta la posibilidad de tener un examen general de orina completamente normal en el escenario de una infección a este nivel, algunos consensos sugieren realizar de forma rutinaria un urocultivo asociado al examen general de orina para descartar o confirmar el diagnóstico ${ }^{2,9}$.

Si se tienen pacientes con diarrea, se debe solicitar directo y cultivo de materia fecal e incluir una prueba para la toxina de Clostridium difficile y examen parasitológico dependiendo de la prevalencia de parasitosis intestinal9,12. Por otro lado, se recomienda tomar muestras de tejido blando ya sea por punción, aspiración o biopsia si la presentación clínica son lesiones nodulares, sin olvidar la posibilidad de una embolia séptica o fungemiaa,9,12. Si el paciente está cursando con una mucositis grado III se ordenan estudios microbiológicos de las mucosas debido a la alta posibilidad de encontrarse ante una lesión por herpes virus o una sobreinfección bacteriana que requiera terapia específica. Por último si se sospecha de esofagitis está indicada la realización de una biopsia de la mucosa para su estudio histológico y microbiológico².

Si el clínico tiene la sospecha que el paciente tiene una infección a nivel del sistema nervioso central, debe solicitar una punción lumbar ${ }^{9,12,23}$. La radiografía de tórax está indicada cuando el paciente presenta síntomas respiratorios con el fin de descartar una 
neumonía ${ }^{12}$, teniendo en cuenta que los hallazgos radiográficos pueden ser inespecíficos y no mostrar las imágenes típicas de consolidación neumónica hasta que haya recuperación del número de neutrófilos ${ }^{9}$, lo cual podría hacer necesario el uso de otras imágenes diagnosticas como la tomografía axial computarizada, que está recomendada en caso de la persistencia de fiebre y la sospecha de foco pulmonar en ausencia de infiltrados en la radiografía.

Si el paciente presenta tos productiva se sugiere hacer Gram y cultivo de esputo ${ }^{2,12}$, mientras que el lavado bronco alveolar es para casos de infiltrados de etiología incierta, persistencia de sintomatología clínica a pesar del manejo instaurado o si hay aparición de infiltrados pulmonares después del séptimo día de tratamiento ${ }^{2,12}$. Entre otros estudios se puede solicitar ecografía abdominal si hay sospecha de tiflitis, y la tomografía computarizada de senos paranasales, abdomen y pelvis se realizarán según lo indique la clínica².

El estudio de una infección fúngica debe considerarse para aquellos pacientes con fiebre persistente 0 recurrente después del cuarto a séptimo día del uso de antibióticos de amplio espectro. En estos casos se recomienda exámenes como fondo de ojo, cultivos, búsqueda de hifas o pseudohifas en orina, biopsia y cultivo de lesiones de piel. En la tomografía de tórax o de senos paranasales se pueden revelar diversas anomalías siendo los macro nódulos con o sin signo del halo los hallazgos más comunes relacionados con aspergilosis invasiva, como también se puede encontrar en esta entidad el signo del aire creciente o lesiones nodulares con cavitación ${ }^{2,9,12}$.

Si se desea detectar Cándida, Aspergillus, Pneumocystis y Fusarium se recomienda pedir el Test Beta-D glucano que tiene alta sensibilidad y especificidad, además su positividad puede preceder los síntomas clínicos de una infección invasiva. Pero si solo se quiere detectar Aspergillus, se sugiere el galactomanano en pacientes con alto riesgo de infección aunque cabe aclarar que hay pobre experiencia en pediatría y puede haber falsos positivos en pacientes que reciben piperacilina tazobactam ${ }^{2,9,12}$.

\section{ESTRATIFICACIÓN DEL RIESGO DE INFECCIÓN BACTERIANA INVASIVA O SIGNIFICATIVA}

El uso de una terapia antimicrobiana empírica de forma temprana ha producido una mejoría en el pronóstico del paciente neutropénico febril, con disminución de la morbimortalidad ${ }^{8,12}$. Para poder realizar un manejo dirigido se han creado modelos predictivos o pronósticos para identificar aquellos pacientes que estarían en alto riesgo de tener complicaciones como sepsis o muerte, y así determinar la necesidad de antibioticoterapia, el tipo de antimicrobiano, la duración y ubicación hospitalaria o ambulatoria del tratamiento.

Con el uso de estos modelos, se pueden determinar aquellos pacientes que se beneficiarían de un manejo menos agresivo, disminuyendo costos y mejorando la calidad de vida del paciente pediátrico con neutropenia febril asociada a enfermedad oncológica. Ninguno de los diferentes modelos predictores ha mostrado supremacía con respecto a otro ${ }^{4}$. Entre los parámetros más frecuentemente usados, se encuentran los propuestos en el estudio realizado en el 2001 por Santolaya y colaboradores 9 , quienes tomaron cinco factores que fueron identificados al ingreso como los que indicaban mayor riesgo de infección bacteriana invasiva y complicaciones.

A pesar de las diferentes propuestas hay dos criterios que se han descrito como los más útiles en el momento de predecir bajo riesgo: el conteo absoluto de monocitos mayor a 100 células $/ \mathrm{mm}^{3} \mathrm{y}$ temperatura menor a $39^{\circ} \mathrm{C}^{4,24}$. Teniendo en cuenta que la fiebre es un signo poco especifíco de infección y que en algunos de los casos obedece a causas diferentes como la enfermedad de base, drogas y transfusiones sanguíneas, las investigaciones recientes han tratado de identificar nuevas herramientas que permitan diferenciar el riesgo de una infección bacteriana.

Debido a la rápida elevación de la procalcitonina en comparación con la proteína $C$ reactiva, se ha propuesto esta como marcador de inflamación $\mathrm{y}$ predictor de riesgo, y se ha demostrado que niveles elevados en el momento del ingreso, principalmente si estos se encuentran por encima de $2 \mathrm{ng} / \mathrm{mL}$, se pueden relacionar con infección bacteriana invasiva en el paciente con neutropenia y fiebre ${ }^{25}$ y puede ayudar a diferenciar la fiebre de causa infecciosa de la no infecciosa ${ }^{26}$. Sin embargo, es importante anotar que el número pequeño de pacientes reportado en estos estudios hace difícil la interpretación de estos resultados. 
Tabla 3. Criterios de alto y bajo riesgo en pacientes pediátricos con neutropenia febril severa ${ }^{9}$

\begin{tabular}{|c|c|}
\hline ALTO RIESGO & BAJO RIESGO 2,7 \\
\hline 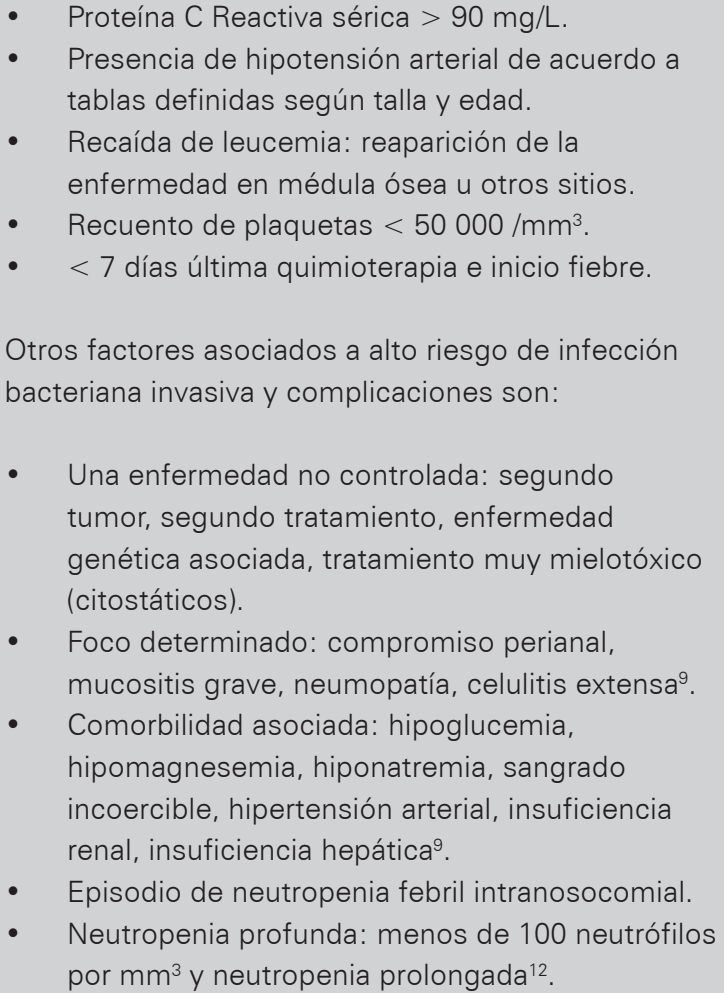 & $\begin{array}{l}\text { - } \quad \text { Proteína C Reactiva baja. } \\
\text { - } \quad \text { Neutropenedad de base en remisión. } \\
\text { de realizada la quimioterapia. } \\
\text { - } \quad \text { Ausencia de hipotensión. } \\
\text { - } \text { Paciente mayor de } 12 \text { meses de edad. } \\
\text { hipomagnesema de los siguientes signos: hipoglucemia, } \\
\text { hipertensión arterial, insuficiencia renal e insuficiencia } \\
\text { hepática. } \\
\text { Episodio de neutropenia febril extra nosocomial. }\end{array}$ \\
\hline
\end{tabular}

Fuente: Autores.

\section{Tratamiento}

La meta de la terapia empírica es iniciar de forma oportuna el manejo antibiótico y así evitar la progresión de una infección y sus complicaciones, hasta determinar un tratamiento dirigido según los resultados de los cultivos. A pesar del uso de antibióticos de amplio espectro, la mortalidad tiene una frecuencia de hasta el $13 \%$ en la mayoría de las publicaciones ${ }^{9,12,27,28}$, por lo tanto se recomienda que todo paciente con fiebre y neutropenia asociada a quimioterapia reciba manejo empírico de forma oportuna, lo cual ha mostrado mejorar el pronóstico, y teniendo en cuenta las posibles infecciones ocultas, se indica mantener la terapia aunque los cultivos sean negativos $^{12,27}$. El tipo de antibiótico para un manejo empírico depende de la clasificación de riesgo del paciente ${ }^{12,29}$.

\section{PACIENTES DE ALTO RIESGO}

Para estas personas se debe ofrecer un manejo intrahospitalario de forma endovenosa. La elección inicial del antibiótico tiene que cumplir una serie de parámetros:

- Cubrir los gérmenes más frecuentemente asociados a infección en pacientes con neutropenia febril, por lo cual las guías recomiendan el uso de antibioticoterapia de amplio espectro en monoterapia o combinación ${ }^{30}$.

- Basarse en los estudios locales de resistencia y el riesgo de inducción de cepas resistentes.

- Debido a la frecuencia de patógenos Gram negativos resistentes, a la mayor mortalidad con Gram negativos que con Gram positivos (mortalidad de $18 \%$ y $5 \%$ respectivamente), y a la asociación con $P$. aeruginosa y las complicaciones que esta produce, se recomienda el uso de un antibiótico dirigido contra estos gérmenes en el manejo empírico inicial ${ }^{27,28}$.

Los protocolos actuales sobre los antibióticos de elección para el manejo empírico inicial sugieren el uso de piperacilina tazobactam, meropenem, 
imipenem o ceftazidima solos o en combinación con un aminoglucosido ${ }^{7,12,30}$. La terapia combinada es superior en ciertas circunstancias, por ejemplo, en infecciones severas ocasionadas por $P$. aeruginosa ${ }^{31}$, sin embargo, las guías indican que ambas aproximaciones tienen resultados similares, con mayor probabilidad de efectos secundarios con la terapia combinada ${ }^{17,28,31,32}$. Por otro lado, la ciprofloxacina está indicada junto con la clindamicina o la vancomicina como alternativas para los pacientes alérgicos a betalactámicos ${ }^{12}$.

La vancomicina no se utiliza de manera rutinaria en el manejo empírico inicial, solo si hay alta sospecha de infección por gérmenes Gram positivos, como ocurre cuando hay inestabilidad hemodinámica, neumonía documentada radiológicamente, hemocultivo positivo para bacterias Gram positivas antes del reporte del antibiograma, sospecha de infección asociada a catéter, infección de piel o tejido blando, colonización con $S$. aureus meticilino resistente o infección por S. viridans ${ }^{7,12,27,29,33}$. No hay beneficio en adicionar empíricamente vancomicina en pacientes neutropénicos con persistencia de fiebre en caso de que se encuentren estables y sin signos de infección (clínica o paraclínica) por gérmenes Gram positivos ${ }^{34}$. Al iniciar terapia con vancomicina esta se debe suspender dos a tres días después si no se ha aislado una bacteria susceptible.

Se recomienda considerar inicio de terapia antifúngica en aquellos pacientes con fiebre persistente 0 recurrente después de cuatro a siete días de manejo antibiótico de amplio espectro si hay inestabilidad clínica asociada o si hay hallazgos paraclínicos que indiquen infección fúngica' ${ }^{12}$. Las levaduras tipo cándida y los mohos como aspergilus y zigomicetos, son los principalmente implicados. Si la profilaxis antifúngica no se ha dado previamente, el riesgo de candidemia es alto, por lo que se puede iniciar con fluconazol; pero si el paciente ya recibe fluconazol, el riesgo de especies resistentes como Cándida Krusei, cándida Glabrata o infección invasiva por moho, es más probable, por lo que se recomienda el inicio de otros agentes como la anfotericina, itraconazol o el voriconazol. El tiempo del tratamiento anti fúngico dependerá de la comprobación de la infección, tipo de hongo localización y estado inmunológico del paciente. Si la infección no se comprueba y el paciente se recupera de la neutropenia puede suspenderse el manejo ${ }^{2,9,12}$.

\section{PACIENTES DE BAJO RIESGO}

El manejo puede ser por vía oral o endovenosa 2,12,10,32,35, por un periodo de observación de 24 a 48 horas en el ambiente hospitalario, en el cual se realizarán estudios para descartar una infección severa, evaluar la estabilidad del paciente y determinar el reporte de los cultivos realizados para así definir la conducta a seguir. En caso de confirmar el bajo riesgo de infección severa y de complicaciones, se optará por un manejo ambulatorio con comunicación diaria con el paciente, siempre y cuando tenga buen acceso a servicios de salud y óptima adherencia al tratamiento. Si la fiebre recurre debe readmitirse e iniciarse el manejo recomendado para pacientes de alto riesgo ${ }^{12,33}$. Las guías recomiendan como terapia endovenosa ceftriaxona con o sin amikacina o la terapia oral con la combinación de ciprofloxacina mas amoxicilina/clavulanato como opción de medicamentos en el manejo de los pacientes con neutropenia febril de bajo riesgo $2,9,12,32,35$. El riesgo de infección fúngica invasiva es bajo, por lo tanto el uso rutinario de terapia antifúngica empírica no está recomendado ${ }^{12}$.

\section{FACTORES DE CRECIMIENTO HEMATOPOYÉTICO}

Los factores de crecimiento hematopoyético regulan la proliferación y diferenciación de las células progenitoras hematopoyéticas y la función de las células sanguíneas maduras, específicamente los factores estimulantes de colonia de granulocitos estimulan la producción de neutrófilos maduros y funcionales. Actualmente hay tres en uso: filgastrim, pegfilgastrim y lenofilgastrim ${ }^{36}$. A pesar de su beneficio en el paciente oncológico, ningún estudio ha demostrado mejoría en la sobrevida con el uso de esta terapia en los pacientes con neutropenia febril, teniendo en cuenta la falta de datos consistentes, los efectos adversos y el alto costo de estos medicamentos. Por tal motivo, hoy en día, no se recomienda su uso como complemento a los antibióticos para pacientes con neutropenia febril ya establecida ${ }^{12}$.

\section{SEGUIMIENTO}

Se recomienda realizar seguimiento paraclínico del paciente al tercer día de iniciada la terapia. En este momento se clasificará como evolución desfavorable, si presenta inestabilidad hemodinámica, aparición 
de un nuevo foco infeccioso o persistencia del foco infeccioso al ingreso, PCR en ascenso $O$ sin disminución significativa (tomando como significativa una reducción de más del 30\% del valor en el reporte anterior), cultivos de control positivos y fiebre persistente sin tendencia a disminuir (más de $38^{\circ} \mathrm{C}$ en dos tomas diarias). Es importante para la evaluación de la persistencia de la fiebre, tener en cuenta que la duración habitual de la fiebre en niños con neutropenia febril de alto riesgo es de cinco a siete días y de dos a tres días en episodios de bajo riesgo ${ }^{2}$.

Posteriormente, la evaluación se realizará cada tres días hasta la resolución del episodio que es considerada como favorable cuando hay un conteo de neutrófilos mayor de 500 células $/ \mathrm{mm}^{3}$ y el paciente tiene más de 48 horas sin fiebre 2 . En caso de que se esté ante un paciente con una evolución desfavorable con pobre respuesta a la terapéutica instaurada, se debe realizar un minucioso examen físico para identificar un foco infeccioso, tomar nuevas muestras de cultivos y exámenes especiales según la sospecha clínica de enfermedad, como ecocardiograma en paciente con catéter venoso central, tomografías, entre otros.

Los antibióticos se deben modificar dependiendo del sitio de infección documentada y el reporte de los cultivos realizados. La fiebre persistente inexplicada en un paciente estable no requiere de cambio de manejo ${ }^{12,28-31}$. Si durante el seguimiento el paciente se encuentra inestable hemodinámicamente a pesar del manejo antibiótico instaurado, se debe ampliar el espectro cubriendo gérmenes Gram positivos, Gram negativos, anaerobios y hongos resistentes ${ }^{12}$. La duración de los antibióticos está guiada por el sitio primario de infección identificado y la recuperación del conteo de neutrófilos. Pacientes con adecuada respuesta a la terapia inicial y con fiebre sin foco se debe mantener el mismo manejo hasta que el paciente esté afebril por dos días y tenga una recuperación medular con neutrófilos mayores de 500 células $/ \mathrm{mm}^{3}$, sin embargo, algunos protocolos recomiendan mantener la terapia por un mínimo de siete días si la evolución es adecuada ${ }^{12,29,32}$.

\section{ConClusiones}

La neutropenia febril en el paciente pediátrico con enfermedad oncológica puede causar una alta morbimortalidad. La mayoría de las veces la fiebre en el paciente neutropénico febril obedece a una infección principalmente bacteriana aunque son diversos microorganismos los que pueden estar implicados. Se deben realizar estudios locales dirigidos a describir los tipos de patógenos, patrones de resistencia y susceptibilidad de los gérmenes involucrados en esta enfermedad con el fin de ayudar al clínico a tomar decisiones en el momento de instaurar una terapia empírica. Debido a que la reacción inflamatoria es escasa en los pacientes oncológicos con neutropenia, es difícil determinar el origen de la infección, por lo que es necesario realizar un examen físico minucioso y usar estudios paraclínicos que ayuden a esclarecer el foco. A pesar que la letalidad en esta patología ha disminuido en la última década, aún sigue existiendo la necesidad de desarrollar mejores métodos diagnósticos y estrategias terapéuticas, así como capacitar permanentemente al personal de la salud para que reconozca de forma temprana esta enfermedad, ya que el diagnóstico oportuno, basados en criterios clínicos y paraclínicos, la categorización del riesgo y el manejo adecuado, según los gérmenes más probablemente implicados, son la clave principal para modificar el pronóstico de esta patología.

\section{ReferenCias Biblografícas}

1. Uribe C, Amado A, Ramírez G, Alarcón I. Cáncer infantil en el área metropolitana de Bucaramanga 2003-2007. MedUNAB. 2011;14(2):86-93.

2. Santolaya M, Rabagliati R, Bidart T, Payá E, Guzmán A, Morales $\mathrm{R}$, et al. Consenso Manejo racional del paciente con cáncer, neutropenia y fiebre. Rev Chil Infect. 2005;22(Supl 2):S79-S113.

3. Gençer S, Slepçi T, Ozer S. Evaluation of infectious etiology and prognostic risk factors of febrile episodes in neutropenic cancer patients. J Infect. 2003; 47(1): 65-72.

4. Phillips B, Wade R, Stewart LA, Sutton AJ. Systematic review and meta-analysis of the discriminatory performance of risk prediction rules in febrile neutropaenic episodes in children and young people. Eur J Cancer. 2010;46(16):2950-64.

5. Matloob M, Fadoo Z. Febrile neutropenia in pediatric cancer patients: Experience from a tertiary health care facility of Pakistan. Pediatr Infect Dis J. 2014;6(3):89-93.

6. Gupta S, Bonilla M, Gamero M, Fuentes SL, Caniza M, Sung L. Microbiology and mortality of pediatric febrile neutropenia in El Salvador. J pediatr Hematol Oncol. 2011;33(4):276-80.

7. Meckler G, Lindemulder S. Fever and neutropenia in pediatric patients with cancer. Emerg Med Clin N Am. 2009;27:525-44.

8. Phillips RS, Sutton AJ, Riley RD, Chisholm, JC, Picton SV, Stewart LA. Predicting infectious complications in neutropenic children and young people with cancer (IPD protocol). Systematic Reviews. 2012;1:8.

9. Comité Nacional de Infectología Pediátrica. Consenso sobre el cuidado del paciente oncológico neutropénico febril. Actualización 2008-2009. Arch Argent Pediatr. 2010;108(2)47-70.

10. Jaramillo C, Valencia IC, Aristizábal MA. Neutropenia febril en pacientes pediátricos: un enfoque diagnóstico y terapéutico. Iatreia. 2009;22(3):235-45.

11. Rueda E, Trujillo ML, Díaz LA. La neutropenia severa febril en niños con cáncer. Estudio descriptivo en el Hospital Universitario de Santander. Salud UIS. 2010;42:103-11.

12. Freifeld AG, Bow EJ, Sepkowitz KA, Boeckh MJ, Ito JI, Mullen $\mathrm{CA}$, et al. Clinical practice guideline for the use of antimicrobial agents in neutropenic patients with cancer. Clinical Infectious 
Diseases. 2011;52:e56-e93.

13. Ke ZY, Xu L, Zhang TT, Mo YL, Huang LB, Zhang XL, et al. A prospective study of febrile episodes in inpatient children on chemotherapy. Pediatr Infect Dis J. 2010;29(10):968-70.

14. Klaassen RJ, Goodman TR, Pham B, Doyle JJ. "Low-risk" prediction rule for pediatric oncology patients presenting with fever and neutropenia. J Clin Oncol. 2000;18(5):1012-9.

15. Ramírez-Londoño LM, Blanco-Villamizar N, Hernández-Escobar M, Sierra-Sánchez M, Aristizábal-Gil MA, Peña-Siado J, et al. Episodios de neutropenia febril en niños con neoplasias malignas. Infectio. 2003;7(3):137-46

16. Basu SK, Fernandez ID, Fisher SG, Asselin BL, Lyman GH. Length of stay and mortality associated with febrile neutropenia among children with cancer. J Clin Oncol. 2005;23(31):7958-66

17. Tezcan G, Kupesiz A, Ozturk F, Ogunc D, Gultekin M, Yesilipek A, et al. Episodes of fever and neutropenia in children with cancer in a tertiary care medical center in Turkey. Pediatr Hematol Oncol. 2006;23(3):217-29.

18. Bakhshi S, Padmanjali KS, Arya LS. Infections in childhood acute lymphoblastic leukemia: an analysis of 222 febrile neutropenic episodes. Pediatr Hematol Oncol. 2008,25(5):385-92.

19. Klaassen IL, de Haas V, van Wijk JA, Kaspers GJ, Bijlsma M, Bökenkamp A. Pyuria is absent during urinary tract infections in neutropenic patients. Pediatr Blood Cancer. 2011;56(5):868-70.

20. Santolaya ME, Farfán MJ, De La Maza V, Cociña M, Santelices F, Alvarez AM, et al. Diagnosis of bacteremia in febrile neutropenic episodes in children with cancer: microbiologic and molecular approach. Pediatr Infect Dis J. 2011;30(11):957-61.

21. Paul M, Gafter-Gvili A, Leibovici L, Bishara J, Levy I, Yaniv I, et al. The epidemiology of bacteremia with febrile neutropenia: experience from a single center, 1988-2004. Isr Med Assoc J. 2007;9(6):424-9.

22. Lucero A Yalda, Brücher U Roberto, Alvarez P Ana María, Becker K Ana, Cofré G José, Enríquez O Nancy, et al. Infección micótica profunda en niños con cáncer, neutropenia y fiebre, en Chile. Rev. méd. Chile. 2002;130(10):1139-46.

23. Urabe A. Clinical features of the neutropenic host: definitions and initial evaluation. Clin Infect Dis. 2004;39(Suppl 1):S53-5.

24. Rackoff WR, Gonin R, Robinson C, Kreissman SG, Breitfeld PB. Predicting the risk of bacteremia in childen with fever and neutropenia. J Clin Oncol. 1996;14(3):919-24

25. Martinez-Albarran M, Perez-Molina Jde J, Gallegos-Castorena S, Sanchez-Zubieta F, Del Toro-Arreola S, Troyo-Sanroman R et a.
Procalcitonin and C-Reactive Protein Serum Levels As Markers of Infection in a Pediatric Population With Febrile Neutropenia and Cancer. Pediatr Hematol Oncol. 2009;26:414-25.

26. Sakr Y, Sponholz C, Tuche F, Brunkhorst F, Reinhart K. The role of procalcitonin in febrile neutropenic patients: review of the literature. Infection. 2008;36(5):396-407.

27. National Institute for Health and Care Excellence. Neutropenic sepsis : prevention and management of neutropenic sepsis in cancer patients. 2012 [Consultado: 04 Oct de 2015]. Disponible en: https:/www.nice.org.uk/guidance/cg151/resources/guidanceneutropenic-sepsis-prevention-and-management-of-neutropenicsepsis-in-cancer-patients-pdf.

28. Glasmacher A, Von Lilienfeld-Toal M, Schulte S, Hahn C, SchmidtWolf IG, Preatice A. An evidence-based evaluation of important aspects of empirical antibiotic therapy in febrile neutropenic patients. Clin Microbiol Infect. 2008;11 Supl 5:17-23.

29. Sharma A, Lokeshwar N. Febrile neutropenia in haematological malignancies. J postgrad med. 2005;51 Supl 1:S42-8.

30. Klastersky JA. Use of imipenem as empirical treatment of febrile neutropenia. Int J Androl. 2003;21(5):393-402.

31. Abhijit M, Gould I. Empirical antimicrobial treatment for chemotherapy-induced febrile neutropenia. International journal of antimicrobial agents. 2007;29(5):501-9.

32. Giurici N, Zanazzo G. Consensus on diagnosis and empiric antibiotic therapy of febrile neutropenia. Pediatric Rep. 2011;3(1):e4.

33. Rivera AN, Boucher HW. Current Concepts in Antimicrobial Therapy Against Select Gram-Positive Organisms : MethicillinResistant Staphylococcus aureus, Penicillin-Resistant Pneumococci, and Vancomycin-Resistant Enterococci. Mayo Clin Proc. 2011;86(12):1230-43.

34. Cometta A, Kern WV, De Bock R, Paesmans M, Vandenbergh M, Crokaert F, et al. Vancomycin versus placebo for treating persistent fever in patients with neutropenic cancer receiving piperacillin-tazobactam monotherapy. Clinical infectious diseases. 2003;37(3):382-89.

35. Freifeld A, Marchigiani D, Walsh $T$, Chanock S, Lewis L, Hiemenz S, et al. A double-blind comparison of empirical oral and intravenous antibiotic therapy for low-risk febrile patients with neutropenia during cancer chemotherapy. N Engl J Med. 1999;341(5):305-11.

36. Welte K, Gabrilove J, Bronchud MH, Platzer E, Morstyn G. Filgrastim (r-metHuG-CSF): the first 10 years. Blood. 1996;88(6):1907-29. 